\title{
Comment écrire l'histoire de la médecine pour les étudiants des sciences de la santé ?
}

\section{Writing down the history of medicine for pedagogical purposes}

\author{
Gaëtan ABSIL ${ }^{1}$ et Patrick GOVERS ${ }^{2}$ \\ 1 Historien et anthropologue, Département des sciences de la santé publique, Laboratoire d'anthropologie sociale et \\ culturelle, Université de Liège, Belgique \\ 2 Historien et anthropologue, Haute École Libre Mosane, Institut Supérieur de traducteurs et d'interprètes, Liège, \\ Belgique
}

Manuscrit soumis à la rédaction le 9 septembre 2014 ; commentaires éditoriaux soumis aux auteurs le 16 mai 2015 ; accepté pour publication le 19 juin 2015

\begin{abstract}
Mots-clés
Histoire ;

médecine ;

enseignement ;

pédagogie

Keywords

History;

medicine;

learning;

pedagogy

Résumé - Contexte : L'histoire de la médecine a acquis une place dans l'enseignement des sciences de la santé. Cet article, écrit par deux historiens travaillant dans le champ de la santé, questionne les manières d'écrire l'histoire de la médecine par des médecins à des fins pédagogiques. Méthode : Les documents $(n=20)$ retenus pour le corpus documentaire sont analysés de manière ethnométhodologique. Résultats : Les auteurs identifient et explicitent sept règles de l'écriture du discours historique dans l'enseignement de l'histoire de la médecine. Les auteurs proposent d'autres règles qui pourraient actualiser les pratiques de l'enseignement de l'histoire dans les sciences de la santé. Conclusion : Se faisant, plusieurs pistes sont proposées pour la construction d'un discours historien porteur de savoir-être et de savoir-devenir. L'objectif est de poser les jalons d'une lecture critique des discours historiques écrits par les professionnels de la santé en mettant en avant le fondement idéologique et mythologique caractérisant l'histoire de la médecine telle qu'élaborée à l'intérieur de ce champ scientifique.

Abstract - Background: The history of medicine is now part of the health sciences curriculum. This article was written by two historians working in the health field. In it, they challenge the ways in which physicians are writing down the history of medicine for pedagogical purposes. Material and method: The corpus of documents $(n=20)$ was analyzed according to ethnomethodological methods. Results: The authors identify eight rules in writing down the history of medicine. They also suggest other rules that could help bring the history of medicine up to date. Conclusions: The objective is to critically review the ideological and mythological premises underpinning the history of medicine today. Further proposals aimed at changing the pedagogical organization of the history of medicine are also suggested.
\end{abstract}




\section{Introduction et problématique : enseigner l'histoire de la médecine}

Comment écrit-on l'histoire de la médecine pour les étudiants en sciences de la santé ? Comment le discours historique est-il composé ? Quelles sont leurs visions de l'histoire ? Quelles sont leurs bibliographies ? Quelle est la finalité de l'histoire de la médecine ? Quelle est la philosophie de l'histoire qui soustend la réécriture par des médecins de l'histoire de leur discipline? À ces questions, plutôt descriptives, s'ajoute une autre série de questions plus critiques. La manière d'écrire l'histoire poursuit des objectifs pédagogiques à la fois explicites et implicites. Les enseignements ont une visée transformatrice et formative. Ce que nous voudrions interroger c'est la manière dont le récit historique est composé à des fins pédagogiques qui participent à la construction de ce discours historique. En quoi ce discours enseigne-t-il un positionnement professionnel qui ne favorise pas le développement d'une compétence culturelle ${ }^{[1]}$ liée à la qualité des soins ?

Un premier état des lieux pointait le retard du développement de l'enseignement de l'histoire de la médecine dans les facultés françaises ${ }^{[2]}$.

Notre point de départ est l'argument suivant : l'enseignement de l'histoire de la médecine, tel qu'il se développe au sein de la plupart des facultés de médecine, est accaparé par les médecins qui en présentent une version essentiellement évènementielle et narrative, d'inspiration globalement « whiggish », qui n'est pas adéquatement exploitée pour susciter chez les étudiants un questionnement épistémologique critique. «Whiggish » désigne une histoire présentée comme marche inévitable du progrès ${ }^{[3]}$.

Cet article est un plaidoyer en faveur de l'intégration des historiens et des techniques historiennes en faculté de médecine et dans l'écriture des manuels d'histoire de la médecine. Il pose la question de la place de l'histoire et des historiens dans l'écriture des manuels. La première partie de ce plaidoyer consiste en une analyse critique des pratiques de l'écriture de l'histoire en faculté de médecine. La deuxième partie, en contre point, propose des pistes " pour mieux faire » à partir des travaux des historiens.
Il ressort de ces deux parties une opposition entre professionnels de la santé et historiens. Cette opposition n'a pas comme objet la revendication (pour une meilleure reconnaissance des professionnels historiens). Elle est une invitation à repenser l'histoire comme une science fondée sur l'enquête.

Cette opposition pourra paraître trop catégorique. Il en va de la volonté des auteurs de proposer un article provoquant le débat et suscitant des réactions auprès des professionnels de la santé.

Afin de comprendre la structuration de cette opposition, il importe d'expliciter, de manière simplifiée, la définition de l'histoire que nous prenons comme référence. Tout d'abord, les deux auteurs, diplômés en histoire de l'Université de Liège, s'inscrivent dans l'histoire sociale et l'histoire culturelle ${ }^{[4-6]}$. Ils ont par ailleurs enseigné l'histoire de la médecine aux étudiants des sciences médicales. L'histoire est une science fondée sur la critique historique et l'analyse des sources. L'histoire sociale étudie les groupes sociaux et l'histoire culturelle peut être définie comme «l'histoire des représentations collectives ». Toutes deux empruntent une partie de leurs concepts à la sociologie et l'anthropologie. Cependant, à côté de cette histoire scientifique existe, mais sans que l'opposition ne soit tranchée, une histoire dite commémorative ${ }^{[7]}$ et identitaire, pratiquée hors des expertises pointues de l'historiographie universitaire.

\section{Méthode}

L'analyse des documents est réalisée sous un angle ethnométhodologique ${ }^{[8-11]}$.L'ethnométhodologie considère les faits sociaux comme des accomplissements pratiques. Elle explicite les savoir-faire, les procédures et les règles de conduite des acteurs dans leurs activités quotidiennes ${ }^{[10]}$. L'enquête ethnométhodologique devrait conduire à élucider les règles de fabrication du discours historique dans les facultés de médecine. Les règles de l'écriture sont présentées dans les résultats selon leur importance pour la structuration du discours. Seules les règles communes à tous les auteurs sont présentées. 
Tableau I. Corpus documentaire des manuels pédagogiques ou des synthèses, utilisés comme ressources didactiques par des professionnels de la santé de langue française pour l'enseignement de l'histoire de la médecine aux étudiants des sciences médicales et paramédicales, au cours des dix dernières années.

Manuels

Ameisen JC, Berche P, Brohard Y. Une histoire de la médecine ou Le souffle d'Hippocrate. Paris : Université Paris Descartes, 2011.

Bertet R. Petite histoire de la médecine. Paris : Editions L'Harmattan, 2005.

Chastel C, Cénac A. Histoire de la médecine : introduction à l'épistémologie. Paris : Ellipses Marketing, 1998.

Ferroul Y, Drizenko A, Boury D. Médecin et médecine : manuel d'introduction à l'étude de l'histoire de la médecine en PCEM 1. Paris : Honoré Champion, 2005.

Fabiani J-N. Ces histoires insolites qui ont fait la médecine. Paris : Plon, 2011.

Gonzalès J. Initiation a l'histoire de la médecine : comprendre pour mieux apprendre. Paris : Heures de France, 1997.

Halioua B. Histoire de la médecine. Paris : Elsevier Masson, 2012.

Hecketsweiler P. Histoire de la médecine, des malades, des médecins, des soins et de l'éthique biomédicale. Paris : Ellipses, 2010.

Magro M. Sous l'œil d'Hippocrate. Petite histoire de la médecine jusqu à nos jours. Paris : Éditions First, 2014.

Meyer P, Triadou P. Leçons d'histoire de la pensée médicale : sciences humaines et sociales en médecine. Paris :

Odile Jacob, 1996.

Dachez R. Histoire de la médecine : De l'Antiquité à nos jours. Paris : Editions Tallandier, 2012

Sournia J-C. Histoire de la médecine. Paris : La découverte, 1997.

Supports de cours

Caubet A. Histoire de la Médecine. Cours, S.L., 2009

Danel V. Petite histoire de la Médecine Occidentale. Cours, Université Joseph Fourier de Grenoble, 2010/2011.

Geenen V. Histoire de la recherche biomédicale. Cours, Université de Liège, 2007.

Gonzalès J, Josset P. Histoire et épistémologie de la Médecine. Cours, CHU- Pitié/Salpêtrière, 2004-2005.

Massart J. Histoire de la médecine. Centre Universitaire de Luxembourg. Département des Sciences, années académique 2001-2002.

L'Histoire de la Médecine http://www.dematice.org/ressources/PCEM1/psychiatrie/P1_psy_007/co/document.pdf

Mairiaux P, Absil G, Govers P. Dimensions psychosociales de la santé. Cours, Université de Liège, 2013.

Neidhardt JPH. Histoire générale de la médecine occidentale, cours, Université de Lyon, sd.

\section{Corpus documentaire}

Nous définissons le corpus documentaire comme la production de manuels pédagogiques, de synthèses utilisées comme supports pédagogiques ou de notes de cours, les dix dernières années, par des professionnels de la santé de langue française pour l'enseignement de l'histoire de la médecine aux étudiants des sciences médicales et paramédicales. La composition du corpus a été réalisée à partir des répertoires bibliographiques et de Google en recourant aux mots clefs suivants : « histoire», «médecine», «soins », «maladie», «médecins ». Cette restriction exclut les histoires consacrées à des pratiques ou des spécialités médicales (chirurgie, obstétrique, génétique, pharmacie). Le corpus documentaire comporte 20 documents (tableau I). 


\section{Résultats}

Règles de l'écriture de l'histoire en faculté de médecine

\section{Règle 1 : Organisation chronologique, diffusionniste et évolutionniste}

Les documents organisent l'histoire de la médecine de manière chronologique. L'histoire commence selon les uns dès la préhistoire, en référence aux travaux des paléontologues, ou tout à fait classiquement avec les civilisations mésopotamiennes. Les auteurs utilisent la division du temps en catégories historiques héritées de la didactique de l'histoire (France et Belgique) pour construire le récit successif de la médecine mésopotamienne, égyptienne, grecque, romaine, médiévale, renaissante, moderne et contemporaine.

L'utilisation des catégories temporelles implique une approche évolutionniste et diffusionniste de l'histoire de la médecine qui naît en Orient puis se diffuse en Occident. Le propos historique se confond avec la visée de vérité, « ce projet d'éclairer d'autres aspects de l'objet, et de nous-mêmes, de situer les illusions et les raisons qui les font surgir, de relier tout cela d'une manière que nous appelons - autre expression mystérieuse - cohérente ${ }^{[12]}$. Nous argumentons que cette visée de vérité tend à soutenir la conclusion « naturelle » que le savoir médical se construit par accumulation pour aboutir à notre époque contemporaine, caractérisée parl'émergence d'un savoir médical institué par les pratiques fondées expérimentalement (evidence based medicine). Le corpus documentaire contient différentes variantes de l'évolutionnisme selon qu'il s'appuie sur la raison, la science, l'écriture, la religion, l'efficacité thérapeutique, etc.

L'histoire de la médecine se présente comme l'archétype de l'histoire du progrès de la science médicale qui, petit à petit, grâce à certains précurseurs, va se débarrasser de toute logique religieuse. Conjointement, le récit est aussi celui d'une profession qui se laïcise et qui se détache du religieux et de la croyance, sources de dogmes et d'erreurs. Le récit reprend la philosophie comtienne des trois âges. L'Antiquité, hormis la parenthèse grecque, se raconte comme âge théologique, la Renaissance comme âge métaphysique et le $19^{\mathrm{e}}$ siècle comme âge positif. Conformément à l'idée hégélienne de l'histoire comme progrès de la raison, les époques intermédiaires (Moyen-âge et époque moderne), sont les antithèses du progrès.

\section{Règle 2 : Valorisation de l'Antiquité, de la Renaissance et du XIXème siècle}

Les premiers chapitres consacrés à l'Antiquité comportent proportionnellement le plus de pages. Alors que les auteurs synthétisent le Moyen-âge en peu de pages, la Renaissance est l'objet d'un développement approfondi. Les derniers chapitres à propos de l'Epoque moderne sont peu épais. Les chapitres retrouvent de l'épaisseur avec le $19^{\mathrm{e}}$ siècle et l'époque contemporaine. La structuration des contenus témoigne de la valorisation de l'Antiquité, de la Renaissance et du XIX ${ }^{\text {ème }}$ siècle. Le Moyen-âge demeure le «dark age » à l'exception de la médecine arabe. L'époque moderne, à l'exception de la Renaissance, n'est qu'une longue stagnation. Cette valorisation des époques est héritée d'une tradition historiographique qui inscrit l'histoire comme le récit du progrès de la raison et des sciences. Dans cette optique, l'histoire de la médecine pourrait se résumer comme l'histoire du miracle grec et de sa continuité jusqu'à ce que les découvertes de Pasteur et l'épistémologie de Claude Bernard prennent le relais, faisant définitivement entrer la médecine dans son régime de rationalité scientifique contemporain.

\section{Règle 3 : Le culturalisme pour relativiser les données contraires au progrès}

Les sociétés antiques et médiévales sont traitées comme des civilisations. Les auteurs résument facilement les sociétés antiques et médiévales à partir de quelques traits. Les chapitres consacrés à l'Antiquité et au Moyen-âge s'inspirent clairement de l'histoire des idées et des religions. L'histoire moderne et contemporaine ayant laissé plus de sources - notamment du fait de l'imprimerie -, les faits sont plus nombreux et les auteurs peinent à trouver une manière de les organiser dans un ensemble culturel cohérent. Il peut arriver que les dernières pages des chapitres sur l'époque contemporaine se transforment en simple 
chronologie, accumulation désorganisée et épreuve de validité du progrès fulgurant et accéléré. Selon le corpus documentaire, l'histoire de la médecine écrite par les médecins est, fréquemment, empreinte d'un rationalisme objectiviste qui se manifeste par la conformation du passé de l'humanité à la raison, c'est-à-dire que tous les faits évoqués possèdent une raison spécifique et que l'ensemble de ces raisons constitue un système cohérent.

Pour les auteurs, il n'est jamais évident d'expliquer les raisons des progrès et des stagnations. Même si cette histoire est celle du progrès dialectique de la pensée médicale, les auteurs ajoutent d'autres arguments qui s'ajustent plus ou moins les uns aux autres. La tension entre changements et permanences participe à la construction d'un discours complexe. Si l'histoire du progrès est celle des découvertes scientifiques, la manière dont ces découvertes sont cadrées par des prémices théoriques peu maîtrisées et héritées des textes de référence conduit à une sorte de répétition du même discours à travers les époques. Elles sont pour la plupart héritées des livres de référence comme par exemple l'évolutionnisme de Lévy-Brul. Elles peuvent être introduites par référence aux travaux des sociologues et des anthropologues. Plus les faits sont éloignés dans le temps, et moins ils ont d'importance pour l'histoire du progrès et plus ils sont interprétés et organisés autour de théories socio-anthropologiques culturalistes. Ces théories implicites ou mal maîtrisées se comportent comme un filtre sur la lecture des événements. Ce filtre conduit à évincer la manière dont l'époque interprétait les événements et conduit à une stabilité des formes dans le temps. D'un point de vue fonctionnaliste, il est quasiment impossible de différencier la fonction de médecin à travers l'histoire. D'un point de vue évolutionniste, la répartition en sciences et croyances reproduit les mêmes partages indépendamment des contextes.

\section{Règle 4 : La Grèce d'Hippocrate comme point de départ de la médecine contemporaine}

Les auteurs reconnaissent Hippocrate comme le fondateur du savoir médical. Ils posent Hippocrate comme la victime de l'Église catholique qui aurait valorisé les écrits de Galien, figeant ainsi le progrès pour plusieurs siècles. Le Moyen-âge, devient l'antithèse d'une médecine humaniste, éclairée et scientifique. Hippocrate, selon les sources documentaires, seraient un Descartes avant-la-lettre en séparant science et religion. Posé comme origine, Hippocrate conjugue rationalité, pratique, éthique et poétique. Argumenter dans ce cadre revient à questionner l'interprétation du moment hippocratique comme un moment de rupture entre l'irrationnel, rupture (de la symétrie) entre l'irrationnel et le relationnel, le rationnel s'imposant peu à peu comme le fil conducteur, le soubassement de notre humanité moderne.

La focalisation des cours sur Hippocrate est exemplative d'une démarche cherchant à établir les origines de la discipline, où la médecine émerge d'entre les sciences pour, par la suite, au travers d'un processus lent, affirmer sa place spécifique dans le champ des sciences. Hippocrate donne ainsi le ton à partir duquel il y aura ou non transcendance du savoir médical quelle que soit l'époque. Le Moyen-âge vaut principalement pour la survie du corps hippocratique, la Renaissance pour l'actualisation de ce même corpus. Pour l'époque contemporaine, Hippocrate devient la bonne mesure contre les « excès » d'une médecine trop technique et plus assez humaniste.

\section{Règle 5 : Identifier les héros et cacher les déviants}

Les figures classiques de la médecine sont avant tout des précurseurs et des innovateurs qui ont su échapper aux entraves que la religion et la croyance font peser sur le progrès du savoir médical. Ils sont reconnus pour leurs idées dès lors que celles-ci s'inscrivent dans la marche du progrès. Ces grandes figures connaissent un double traitement. Elles sont traduites une première fois de manière anachronique où toute référence à ce qui apparait comme religieux est expurgée des sources. Elles sont traduites une deuxième fois pour une intégration dans un grand récit du progrès. La biographie de Paracelse, par exemple, se voit réduite à celle d'un précurseur de la pharmacie moderne et de la médecine moléculaire. Le système des éléments et leurs correspondances zodiacales sont évacués. Paracelse cesse d'être le Paracelse historique pour devenir un Paracelse de convention. Semmelweis est utilisé comme figure du précurseur martyr. Elle sert 
et commémore le propos d'un progrès entravé par les croyances et libéré par des héros. S'il est aisé de repérer la figure du héros et celle du martyr, le corpus est plus avare lorsqu'il s' agit de médecins qui ne sont pas vecteurs de progrès ou qui auraient trahi l'idéal humaniste. La figure de l'eugéniste d'Alexis Carrel n' apparaît pas dans le corpus documentaire.

Le récit esquive les erreurs de la médecine dès lors qu'elles ont des conséquences considérées «mauvaises » sur un plan axiologique. La participation de la médecine à la raciologie, au racisme ou à l'expérimentation sur les êtres humains ${ }^{[13]}$ n'est pas à l'ordre de l'enseignement. Lorsqu'un courant médical se voit critiqué, la critique est atténuée par l'argument de l'esprit du temps. Ainsi, l'hygiénisme sert facilement d'exemple pour un progrès sociétal dû à la médecine et que les auteurs critiquent, parfois, pour son racisme social. Or, ce moment où la médecine participe à l'institutionnalisation de l'ingénierie sociale ${ }^{[14]}$ dans la foulée de l'hygiénisme devient un repoussoir qui n'est pas interrogé dans ses conséquences. Les excès sont plus facilement attribués à la civilisation ou à une école de pensée présentée comme définitivement passée. Les filiations s'établissent plus facilement avec les héros et les martyrs, tandis que les déviants sont isolés dans une séquence culturelle définitivement passée.

\section{Règle 6 : Expliquer l'état présent comme aboutissement logique du passé}

L'histoire de la médecine part d'un anachronisme. Pour définir la médecine, les auteurs utilisent trois dimensions : la théorie, la pratique et la professionnalisation. Ainsi, les cours explorent-ils pour chaque époque ce qui relève des théories explicatives de santé et des maladies, des pratiques préventive et curative et des professions impliquées dans le soin. L'usage de ces trois dimensions prend la forme d'une recherche du même à travers l'histoire. Les évènements retenus pour les cours sont finalement ceux qui démontrent l'existence d'une pensée organisée dans laquelle sont en germes les prémices de notre propre fondement logico-ontologique, de pratiques exotiques par leurs audaces en des temps archaïques et de l'ébauche de l'institutionnalisation des professions de la santé. Ces trois dimensions ont pour effets de disqualifier du savoir médical en construction toutes une série d'acteurs et de théories. Elles écrivent une histoire qui reproduit des dominations symboliques d'un savoir masculin sur un savoir féminin, d'un savoir laïc sur un savoir religieux, d'un savoir institué sur un savoir profane. Tous les savoirs et les acteurs qui ne sont pas à l'heure actuelle reconnus par la faculté comme des acteurs légitimes de la santé tendent à être évacués, par exemple la figure de la sage-femme associée à la sorcellerie au bas moyen âge. Les sages-femmes accusées de sorcellerie sont présentées comme des criminelles : elles tuent, elles empoisonnent, elles commettent des crimes sexuels et des conspirations (aider et soigner). Le fait que les hommes des classes supérieures soignent sous l'auspice de l'église ne pose pas problème, le fait que les femmes provenant de la classe paysanne soignent en est un ${ }^{[15]}$. Cette disqualification paraît très contradictoire. Les travaux des sociologues et des anthropologues montrent clairement que le soin se réalise par réseaux thérapeutiques correspondant à une étiologie de facteurs individuels, naturels, sociaux et surnaturels ${ }^{[16]}$.

Les auteurs traitent les biographies et les citations comme les humanistes ont traité les philosophes antiques, c'est-à-dire en reconnaissant leur intérêt tout en reconnaissant leurs erreurs, l'erreur n'étant pas pour la médecine, la reconnaissance de Dieu à venir, mais bien celle d'une science à venir. Comme Dante plaçait les philosophes et savants grecs dans le purgatoire, l'histoire de la médecine place les mêmes savants dans une préscience du futur. Ceci constitue le biais d'interprétation dit «a posteriori » qui présume que les acteurs ont conscience des conséquences historiques de leurs actions ou des changements sociaux.

\section{Règle 7 : La finalité pédagogique pour justifier les simplifications}

La didactique semble pensée sur la règle « on ne forme pas des historiens ». Il s'agit donc d'offrir une vision simplifiée et logique de l'histoire. Cette simplification implique un renoncement à une version scientifique de l'histoire fondée sur la critique historique $^{[17]}$, qui ne confond pas fait et évènement, qui n'est pas téléologique, qui connait son historiographie, qui fait appel aux méthodes actualisées de la discipline 
historique $^{[6,18]}$. Lorsqu'il faut choisir des contenus, la question incontournable est celle de l'utilité du contenu pour les étudiants : un contenu qui peut être compris sans référence aux travaux des historiens, qui peut s'expliquer en référence à nos propres connaissances, qui valorise la pensée médicale au fil du temps.

La didactique utilise des citations pour exemplifier les propos des cours. Les citations mobilisent des auteurs célèbres. Elles sont traitées par un processus de décontextualisation et recontextualisation qui oblitère le contexte culturel de sa production. Ainsi, les mots sont-ils interprétés avec nos propres significations. Les textes de référence sont amputés de parties qui ne servent pas le propos du récit. Pour Vésale, par exemple, les citations seront uniquement celles qui indiquent un progrès. Les citations sont présentées comme la vérité de notre époque en gestation et non encore révélée par les lumières de la science.

Les histoires sont principalement écrites à partir des travaux de seconde main, qui synthétisent d'autres travaux. Si les travaux de seconde main peuvent être dévalorisés dans le champ de la recherche historique, il est plus difficile de critiquer l'intérêt de l'existence de synthèse face à un savoir éparpillé dans les revues et les monographies. Lorsqu' on analyse les bibliographies utilisées ou mentionnées par les auteurs, toutes les écoles historiques ne sont pas représentées de la même manière. Les auteurs s'entre-citent, tant et si bien qu'il est possible d'identifier les quelques ouvrages de références qui structurent les récits et par rapport auxquels les autres ouvrages en sont des actualisations. Sans qu'il s'agisse de remettre en question la valeur ou l'intérêt des monographies canoniques, nous observons ici leur utilisation pratique. Une fois que l'usage et le crédit de l'auteur ont passé les épreuves de validation, ces synthèses deviennent incontournables car, d' un point de vue strictement pratique, il est plus rentable de rédiger un cours sur base d'une synthèse.

\section{De nouvelles règles}

A la lecture des documents, il apparaît que l'histoire écrite par les professionnels de la santé pourrait s'apparenter à une histoire d'amateurs éclairés à des fins symboliques et pédagogiques, dont l'épistémologie réside dans les sciences médicales. Ce récit historique a tout du mythe ${ }^{[19]}$. Les récits se ressemblent, les auteurs reprennent sans cesse le même canevas, les mêmes figures à quelques détails près. Il serait tout à fait juste de considérer l'ensemble du corpus comme un seul récit et ses variations. Ce récit vise à permettre aux étudiants de partager une vision commune de l'histoire de leur science qui est aussi un discours idéologique. En ce sens, en promouvant à travers les cours une version établie de l'histoire, les auteurs limitent l'ouverture des étudiants vers d'autres versions de l'histoire des médecines, qui paraissent, par contraste, plus critiques et moins complaisantes.

\section{Règle $A$ : Utiliser un cadre théorique clair}

Ces discours manquent d'un cadre théorique qui agencerait les contenus. L'agencement selon les catégories temporelles de la didactique induisent des difficultés pédagogiques ${ }^{[17]}$. Ces difficultés concernent par exemple l'approche de la complexité que représentent les enchevêtrements d'objets tels que la santé, la maladie, le corps, les soins, la mort, la vie, l'alimentation, la religion, de tous ces objets qui, même d'un point de vue anachronique, participent de la santé au sens large et qui ont leurs propres temporalités : le découpage selon les périodes complique plus qu'il ne clarifie le $\operatorname{propos}^{[20]}$. La théorie des humeurs, par exemple, traverse l'histoire de la médecine jusqu'au début du XX ${ }^{\text {ème }}$ au moins. D'un point de vue pédagogique une vision encyclopédique de l'histoire de la médecine pourrait être abandonnée, pour une histoire problématisée, pourquoi pas rétrospective, ou qui s'accommoderait d'une périodisation moins classique.

Les auteurs assemblent des explications issues de plusieurs théories sociologiques, anthropologiques et historiques. Le plus souvent, ils reprennent les explications des anthropologues pour les aspects culturels des sociétés archaïques. Or, les théories anthropologiques prétendent à une visée universelle et devraient valoir pour toutes les cultures et civilisations. Cette caractéristique rend le discours incohérent. Par exemple, la maladie est une fatalité et une punition des dieux pour l'époque archaïque, et ce raisonnement n'est pas repris pour les périodes suivantes, ni même 
pour l'époque contemporaine, ce qui est contraire aux recherches en anthropologie de la santéf ${ }^{21]}$.

\section{Règle B : Questionner l'utilité d'une histoire depuis les origines}

Qu'il soit possible d'identifier des pratiques de soins et de prévention dans toutes les civilisations depuis la «nuit des temps » ne signifie pas que les origines doivent être enseignées. Au fond, il s'agirait de démontrer par accumulation des faits, mais sans tenir compte que ces faits sont eux-mêmes une construction, qu'il y a toujours eu une médecine. Autrement dit, la médecine serait consubstantielle à toute forme de civilisation, elle en serait un invariant. Cette conclusion, qui pourrait être enseignée, témoigne d'un discours à forte intonation fonctionnaliste, selon lequel toute activité humaine serait une réponse à des besoins naturels, la fonction de la médecine étant de préserver la vie. Nous pourrions inverser le raisonnement et nous demander si ce n'est pas l'importance de la médecine dans les sociétés occidentales qui construit ce trait de civilisation.

L'origine hippocratique est plutôt rentable. Hippocrate permet de poser les fondements idéaux de la médecine. La figure d'Hippocrate devient une forme « pure » et « simple » pour comprendre l'institution médicale, tout comme il est d'usage d'étudier la démocratie grecque comme forme pure. Il y a une différence à poser Hippocrate comme cas, dans le cadre d'une problématique historique, et à le poser comme commencement.

\section{Règle $C$ : Plus d'historicité, moins d'interprétation "a posteriori "}

S'il est vrai qu'aucune société ne peut survivre sans organiser sa production matérielle et sa reproduction, Castoriadis ${ }^{[12]}$ pointe le fait que cette organisation n'est conduite ni par la rationalité pure ni par des lois naturelles. Cette réflexion de Castoriadis nous rappelle qu'il est important d'objectiver la visée de vérité qui guide le discours historique de la médecine écrit par des professionnels de santé ${ }^{[22]}$, comme en témoigne la déstructuration des textes au profit de l'accumulation d'évènements dans les chapitres consacrés à l'histoire contemporaine.
À cette fin, l'historicité pourrait être développée par les auteurs de manuels en abordant la pluralité des formes du soin dans un contexte socioculturel donné. Qu'est-ce qui constitue le soin dans une société spécifique à un moment précis de son existence ? Comment est-il organisé ? Comment est-il perçu et vécu par les différents acteurs en présence ? Comment est-il communiqué, inscrit, traduit (mis en discours) ?

Les manuels anglo-saxons d' anthropologie médicale ont pour objectif de développer une compétence culturelle chez les étudiants. Pourquoi ne pas reprendre cet objectif au compte de l'enseignement de l'histoire de la médecine ? Il ne s'agirait pas de dire que l'on est en opposition avec ce qui est considéré comme des erreurs du passé, mais plutôt de reconnaître et d'identifier les formes et les lieux du discours médical, sans a priori définir celui-ci selon son efficacité et son régime de vérité actuel. Il devient alors difficile de reconnaître que le recours à une amulette puisse protéger de la peste, que le culte des saints puisse constituer une forme de soin ou une forme de santé publique. Les jugements portés sur ces évènements sont aussi une manière de stigmatiser les pratiques de médecines non conventionnelles. Le jugement des auteurs sur l'inefficacité du culte des saints vaut pour hier et pour aujourd'hui, niant son importance culturelle ou psychologique pour les croyants. Si ce jugement est recevable du point de vue biomédical, il l'est moins du point de vue socio anthropologique. Ce type de jugements construirait, selon nous, un positionnement professionnel implicite chez les étudiants qui valorise la médecine scientifique et rend difficilement concevable d'autres modes d'efficacité. Le savoir médical dit la vérité d'un monde ramené à une dimension biologique perçue comme invariante : le corps du pharaon, du chevalier, de l'humaniste sont biologiquement identiques à celui de patients de notre époque. Ce positionnement entrave toute possibilité de relativisme qui ne serait pas un mépris déguisé. Ce positionnement entrave la possibilité d'une compréhension de l'autre, celle-ci étant pourtant constitutive de la qualité des soins. Ce projet d'élucidation pour des futurs professionnels de santé peut s'avérer l'occasion de redire, et par la même, renforcer la vision complexifiée du fait humain. Il s'agirait de développer une pratique pédagogique qui s'appuierait sur un savoir, non 
pas présenté comme total et atemporel, mais davantage comme fragmentaire et provisoire. Fragmentaire parce qu'il n'existe pas de théorie exhaustive de l'homme et de l'histoire, provisoire car la pratique doit favoriser l'émergence d'un nouveau savoir dans la perspective d'une pédagogie pragmatique fondée sur l'expérience.

\section{Règle $D$ : Un renouvellement de la bibliographie}

L'historiographie médicale se renouvelle ${ }^{[23-31]}$. Les auteurs des cours devraient pouvoir s'approprier le propos historique de ces monographies et articles. Lorsqu'un évènement historique est inséré par les historiens dans un cadre ou système d'analyse cohérent, les auteurs des manuels ne retiennent que l'évènement lui-même en l'extrayant de son cadre d'interprétation. Les auteurs du corpus documentaire n'utilisent jamais la perspective historique des ouvrages de Canguilhem ${ }^{[32]}$, d'Elias ${ }^{[33]}$ ou de Foucault ${ }^{[34,35]}$. La proposition de Foucault d'une médecine dont les normes se construisent à partir du pathologique et de la mort, pourrait être un levier pédagogique intéressant. D'autres auteurs pourraient être remis à l'ordre du jour pour une autre histoire de la médecine, par exemple, les travaux de Lichtenthaeler ${ }^{[36]}$ et la synthèse de Starobinsky ${ }^{[37]}$, tous deux médecins. Ces deux références sont intéressantes car elles proposent un programme d'histoire de la médecine dégagée de sa vision positiviste. Outre ces auteurs devenus des classiques, nous pouvons mentionner pour les travaux francophones ceux de Corbin ${ }^{[27]}$, Faure ${ }^{[25]}$, Goubert ${ }^{[29,30]}$, Grmek $^{[38]}$, Havelange ${ }^{[39]}$, Mandressi ${ }^{[26]}$, Sendrail ${ }^{[40]}$, par exemple pour se limiter à la médecine. L'historiographie anglosaxonne renouvelle aussi le regard sur la médecine avec, par exemple, les travaux de Roy Porter $^{[41-44]}$, Dorthy Porter ${ }^{[45,46]}$ et Dufin ${ }^{[47]}$. Une explication de la plus-value pédagogique de ces travaux constituerait un article en soi. À la décharge des auteurs du corpus, il n'existe pas de synthèse récente de l'histoire de la médecine écrite par un historien, suite vraisemblablement à l'abandon des histoires totales et à la dévalorisation des synthèses dans l'évaluation des publications. De plus, l'émiettement des objets historiques augmente considérablement la difficulté d'en réaliser une synthèse ${ }^{[48]}$.

\section{Règle $E$ : L'interdisciplinarité}

L'intégration ou l'attachement d'historiens dans les facultés de médecine pourrait être une piste, de même que celle des médecins dans les unités d'histoire de la médecine. Cependant, cette piste devra être pensée en relation avec plusieurs obstacles. D'une part, la main mise par les médecins sur l'enseignement de l'histoire en faculté de médecine questionne l'interdisciplinarité : pour parler de médecine, il faut être médecin. Cet obstacle est d' autant plus difficile à lever que l'histoire de la médecine s'écrit en référence à une visée de vérité qui sous-tend l'histoire de la médecine écrite par les professionnels de santé. D'autre part, les historiens ont fort à faire pour mieux comprendre les logiques du discours médical afin d'instaurer un dialogue avec les professionnels de santé. Ce manque de reconnaissance, cette guerre d'experts, constitue l'obstacle majeur pour établir des ponts entre les disciplines médicale et historique : deux disciplines qui relèvent d'un savoir expert et qui peuvent être en concurrence de par leur prestige symbolique, leur forte institutionnalisation et une tentative de dominer leurs objets respectifs ${ }^{[49]}$.

\section{Règle F : Une problématisation de l'enquête}

L'enseignement de l'histoire peut prendre la forme d'une enquête au sens de Dewey ${ }^{[50]}$. L'enquête commence par un questionnement et, dans ce cadre, il paraît opportun que le questionnement noue histoire et enjeux actuels. La problématique structure le récit autour d'objets plus précisément définis que ne peut l'être histoire de la médecine ${ }^{[51]}$. La problématisation réalise un lien entre le présent et le passé, qui ne consiste pas à rechercher les fondations mais à comprendre comment la médecine s'exerce au présent. $\mathrm{Ce}$ lien crée une distanciation par le moyen d'un propos historique à des fins pédagogiques.

Au lieu de présenter Hippocrate comme le père de la médecine, posons la question : Hippocrate peut-il être considéré comme le père de la médecine et tentons d'y répondre avec les étudiants. D'autres sujets d'enquête peuvent être facilement conçus. Par exemple, la figure d'Hildegarde de Bingen permet à la fois d'enquêter sur la médecine médiévale dans 
toutes ses dimensions tout en enquêtant sur des enjeux actuels comme le recours aux médecines alternatives ou sur la place des femmes dans la profession médicale. Le travail de Duffin ${ }^{[47]}$ montre qu'il est possible d'investiguer sur la réalité et la définition des maladies à travers le temps.

\section{Règle G : Considérer l'histoire comme un savoir scientifique}

Les sciences médicales reposent de plus en plus sur l'evidence based comme la forme exigeante de scientificité ${ }^{[52]}$. Pourtant les écrits du corpus respectent peu les critères scientifiques propres à l'histoire. La rationalité scientifique médicale imprime l'organisation et la finalité du discours. Elle n'imprime pas la méthode de rédaction du récit qui ne repose jamais sur une enquête inductive ou déductive. Il est très différent, respectivement, d'inscrire la médecine du Moyen-âge dans la croyance au fil d'un récit évolutionniste ou de poser la question : « la médecine du Moyen-âge estelle uniquement fondée sur la croyance ?». Comment expliquer ce paradoxe ? L'histoire ne serait pas considérée comme une science par les médecins. Cette non reconnaissance autorise une liberté par rapport aux contenus et à la méthode. La finalité pédagogique et mythologique inviterait à une instrumentalisation idéologique de l'histoire. Faudrait-il rappeler que l'histoire procède aussi de raisonnements scientifiques de types déductifs ou inductifs?

\section{Règle $H$ : Une histoire qui est aussi celle des malades et des patients}

L'histoire de la médecine se résume à celle des grands hommes et de leurs idées ou à une histoire des maladies et des remèdes. Rarement, les historiens médecins font-ils une histoire de la santé ou une histoire des malades. La présence des malades doit tout à la présence du médecin dont ils sont les objets ${ }^{[53]}$. Les corps malades enregistrent les succès de la médecine et les échecs des croyances. Les malades ne sont envisagés qu'à travers leur rôle dans le colloque singulier alors qu'ils pourraient être étudiés en dehors de leur lien avec les soignants. L'histoire de la médecine écrite par les médecins n'échapperait donc pas au socio-centrisme ${ }^{[12]}$, à cette façon d'afficher une visée de vérité propre à des professionnels de la santé, membres d'une classe professionnelle dominante défendant une position stratégique à l'intérieur d'un champ déterminé. Par exemple, le rôle des corporations dans l'organisation des pèlerinages thérapeutiques pourrait être rapproché de celui des associations de patients, questionnant le rôle de malade dans la gestion sociale de la maladie ${ }^{[54,55]}$. À cet égard, le développement des théories de l'embodiment ${ }^{[56]}$ représente une opportunité pour aller au-delà ; une invitation à renouveler le regard critique, à nous recentrer davantage sur le corps du malade comme lieu multiple d'expérience de la maladie, de la santé et de la médecine ${ }^{[57]}$.

En lien avec un renouvellement de bibliographie, l' " histoire des patients », comme elle est développée depuis les années 80 et l'article séminal de l'historien britannique Roy Porter ${ }^{[42]}$, est aussi une piste à suivre. Ce courant s'est surtout attaché à rendre aux malades dans l'histoire leur capacité d'action, notamment en les positionnant comme des acteurs cruciaux du champ médical et en étudiant l'impact des choix qu'ils opèrent dans ce contexte. Cette histoire, toute entière consacrée à l'étude des souffrants, se penche d'ailleurs souvent sur la relation médecin-malade, relation qu'elle historicise et ouvre à l'individualité et la subjectivité du malade.

\section{Discussion}

Nos références théoriques empruntent aux courants de l'histoire sociale et culturelle ${ }^{[5,6,39,58,59]}$. Il $\mathrm{y}$ a une difficulté méthodologique à prendre l'histoire culturelle comme référence. Cela revient à choisir comme norme d'évaluation celle qui, a priori, pourrait être la plus éloignée d'une pratique normale de l'histoire, celle qui procède de l'histoire, dite méthodique. Nous mettrions d'emblée en opposition une histoire culturelle centrée sur les questions de médiations symboliques ${ }^{[60]}$ avec une histoire méthodique où l'accumulation des faits avérés fait récit. Nous avons peu d'indice de l'adhésion explicite des auteurs à une école historique. 
Ce que l'article analyse, ce sont les pratiques d'écriture de l'histoire des auteurs de 20 ouvrages/ manuels destinés aux étudiants des sciences de la santé. Ce corpus ne nous permet pas de généraliser les analyses et les conclusions à l'ensemble de la production historique signée par les professionnels de la santé, ce qui serait une autre enquête. Il n'est d'ailleurs pas difficile de trouver des contre-exemples en termes d'écriture historique par ces mêmes professionnels de la santé (Ackerknecht, Temkin, Sigerist, Sendrail). Evitons donc d'étendre notre questionnement en dehors du champ des pratiques pédagogiques.

Notre article se limite à la manière d'écrire l'histoire et à ses implication pédagogiques pour la formation des étudiants. Notre analyse gagnerait en pertinence si elle était complétée par une enquête par entretiens auprès des auteurs afin de mieux comprendre et expliciter leurs intentions et pratiques.

\section{Conclusion}

Il y a une pratique historienne propre aux professionnels de la santé qui n'est pas celle des historiens, qui n'est pas vraiment celle des amateurs ou des membres des sociétés historiques locales. Il s'agit d'une forme hybride de discours, qui arrange les événements selon un ordre soutenu par le réductionnisme et l'humanisme ${ }^{[16]}$. Ce phénomène d'appropriation d'un champ du savoir par la médecine ne se limite pas à l'histoire. Les méthodologies, pourtant bien définies par les praticiens des sciences sociales et humaines, lorsqu'elles sont utilisées par les sciences médicales, deviennent stéréotypées tant au niveau théorique que pratique $^{[61]}$. Les sciences médicales les dotent d'un nouveau régime de vérité, elles retiennent ce qui fonctionne, ce qui cadre avec une médecine fondée sur les preuves $^{[62]}$, c'est-à-dire sur des résultats exempts d'incertitude. Or, « la tâche première de l'historien comme de l'ethnologue est donc de retrouver ces représentations dans leur irréductible spécificité, sans les recouvrir avec des catégories anachroniques, ni les mesurer à l'aune de l'outillage mental du XX $\mathrm{X}^{\mathrm{èm}}$ siècle, posé comme le résultat nécessaire d'un progrès continu ». ${ }^{[63]}$
«Le faire de l'histoire s'arc-boute sur un pouvoir politique qui crée un lieu propre (cité, nation, etc.), où un vouloir peut et doit écrire (construire) un système (une raison articulant des pratiques) » ${ }^{[22]}$. Le discours historique des médecins crée la médecine comme lieu d'un savoir vrai et efficace sur la santé et la maladie. La volonté des auteurs construit un système articulé sur l'histoire du progrès et de la raison par la mise en relation d'un corps professionnel et de ses pratiques. Ce lieu isole à travers le temps une version biomédicale de la santé et de la maladie par un processus d'exclusion de l'altérité, constituée sur le socle des erreurs et des égarements des croyances. La médecine apparait comme une science dont le développement fut lent, tant il fut freiné par la prévalence de galénisme et de la religion, et qui ne prend toute sa mesure qu'au moment de la médecine expérimentale de Claude Bernard. Le choix de cette césure avec une médecine du passé, c'est-à-dire une médecine liée à l'irrationalité, est présenté comme un aboutissement logique. Il n'y a pas de remise en perspective, ni épistémologique (on ne questionne pas le paradigme qui sous-tend le régime de vérité du discours médical occidental qui se forge à la fin du XIX ${ }^{\text {ème }}$ siècle), ni idéologique (on laisse dans l'ombre les rapports de force qui traversent tout récit) ${ }^{[64]}$.

L'histoire de la médecine au carrefour ?

L'identité professionnelle se constitue entre autre en référence à une histoire commune qui explique et qui légitime la place des professions dans la société. L'histoire, sous forme de récit, enseignée aux étudiants en sciences de la santé, est différente d'une histoire fondée sur la critique historique. Elle constitue néanmoins l'une des versions de l'histoire en tant que discours pluriel et pratique sociale. Dénier tout intérêt ou légitimité à cette pratique historienne reviendrait à ne plus prendre le risque d'une histoire au carrefour des disciplines ${ }^{[65,66]}$, à condition que ce carrefour soit bien un croisement interdisciplinaire ${ }^{[67]}$ et non la priorité d'une discipline sur l'autre. Il serait utile de réfléchir au sens de l'histoire qui est promue auprès des étudiants.

Une caractéristique joint l'histoire et la médecine. Elles peuvent être vues comme des savoirs de carrefour ne pouvant se constituer sans l'apport d'autres 
champs du savoir. Les sciences humaines interrogent le savoir médical et l'empêchent de s'enfermer dans le champ de la physique, de la chimie ou de la biologie.

Il convient, selon nous, de ne pas perdre de vue que les sciences sociales attachent une grande importance au développement de la pensée critique. Celle-ci se compose de deux éléments : la curiosité (désir de comprendre) et l'indignation (la volonté de transformer) ${ }^{[68]}$. Elle met en avant un regard sur le présent - conceptualisé comme moment historique - et l'importance de l'engagement, c'est-à-dire la capacité d'étonnement. En d'autres termes, la pensée critique nous rappelle qu'il est impératif de replacer tout discours dans son contexte socio-anthropologique d'énonciation ainsi que d'en établir une généalogie, sans quoi le risque est grand que ce discours ne se transforme en interprétation unique pour appréhender toute réalité humaine.

Le temps n'est-il pas venu de remettre en question cette visée de vérité élaborée par des professionnels de santé à des fins pédagogiques, de renouveler la critique sur la base d'une perspective focalisant simultanément l'engagement et la distanciation ? La critique nous invite à prendre en compte la complexité et l'indétermination des intentions et des actions humaines, et permet de questionner ce besoin existentiel de donner du sens (récit, histoire, fiction) à ce que nous faisons. Point de départ pour la construction d'un savoir interdisciplinaire critique?

\section{Contributions}

Les deux auteurs ont contribué également à ce travail (problématique, collecte des documents, analyse, rédaction).

\section{Déclaration d'intérêts}

Les auteurs ne déclarent aucun conflit d'intérêt en lien avec le contenu de cet article.

\section{Approbation éthique}

Non sollicitée car sans objet.

\section{Références}

1. Winkelman M. Culture and health: Applying medical anthropology. New York: John Wiley \& Sons, 2008.

2. Bouchet A, Charlier P. L'enseignement de l'histoire de la médecine dans les institutions universitaires françaises. Histoire des sciences médicales 2008;42:145-8.

3. Alvargonzález D. Is the history of science essentially whiggish? History of Science 2013;51:85-100.

4. Le Goff J, Nora P. Faire de l'histoire: nouveaux problèmes, nouvelles approches, nouveaux objets. Paris : Gallimard, 2011.

5. Burke P. What is cultural history. New York: Polity, 2008.

6. Ory P. L'histoire culturelle. Paris : Presses universitaires de France, 2004.

7. Bourdieu P. Sur les rapports entre la sociologie et l'histoire en Allemagne et en France. Actes de la recherche en sciences sociales 1995;106:108-22.

8. Garfinkel H, Quéré L. Recherches en ethnométhodologie. Paris : Presses universitaires de France, 2007.

9. De Fornel M, Ogien A, Quéré L. L'ethnométhodologie : une sociologie radicale. Paris : Editions La Découverte, 2000.

10. Coulon A. L'ethnométhodologie. Paris : Presses universitaires de France, 1987.

11. Heritage J. Ethnomethodology. In: Giddens A, Turne J. (Eds.). Social Theory Today. Cambridge: Polity Press, 1987:224-72.

12. Castoriadis C. L'institution imaginaire de la société. Paris : Seuil, 1975.

13. Chamayou G. Les corps vils : expérimenter sur les êtres humains aux XVIII ${ }^{\mathrm{e}}$ et $\mathrm{XIX}^{\mathrm{e}}$ siècles. Paris : $\mathrm{La}$ Découverte, 2008.

14. Havelange C. La Belgique médico-sociale. L'individu, la société, la race. In: Kurgan van Hentenriyk G. (Ed.) Laboratoires et réseaux de diffusion des idées en Belgique (XIX ${ }^{\mathrm{e}}-\mathrm{XX}^{\mathrm{e}}$ siècles) Bruxelles : Editions de l'Université Libre de Bruxelles, 1994:19-33.

15. Ehrenreich B, English D. Witches, Midwives, and Nurses: A History of Women Healers. New York: Feminist Press, 2010.

16. Helman CG. Culture, health and illness: An introduction for health professionals. Oxford: Butterworth-Heinemann, 2014. 
17. Halkin L. Initiation à la critique historique. Liège : Har, 1988.

18. Pomian K. Sur l'histoire. Paris : Gallimard, 1999.

19. Absil G, Govers P, Santerre H, Fond-Harmant L. Comment utiliser les mythes pour construire un discours sur la santé mentale. In : Valastro OM. (Ed.). Mythanalyses postmodernes de la santé mentale. Les cahiers de M@gm@2014;7:193-210.

20. Le Moigne J-L. Les épistémologies constructivistes. Paris : Presses Universitaires de France, 1995.

21. Augé M, Herzlich C. Le sens du mal : anthropologie, histoire, sociologie de la maladie. Paris : Editions des Archives contemporaines, 1983.

22. De Certeau M. L'écriture de l'histoire. Paris : Gallimard, 1975.

23. Gourevitch D. Histoire de la médecine. Annuaire de l'École pratique des hautes études (EPHE), Section des sciences historiques et philologiques Résumés des conférences et travaux. 2011(141):300-301.

24. Faure O. Les Français et leur médecine au XIX ${ }^{\mathrm{e}}$ siècle. Paris : Belin, 1993.

25. Faure O. Histoire sociale de la médecine. Paris : Economica, 1994.

26. Mandressi R. Le regard de l'anatomiste : dissections et invention du corps en Occident. Paris : Seuil, 2010.

27. Corbin A. Le miasme et la jonquille. Paris : Editions Flammarion, 2010.

28. Corbin A. Les filles des noces. Paris : Aubier Montagne, 1978.

29. Goubert J-P. Initiation à une nouvelle histoire de la médecine. Paris : Ellipses, 1998.

30. Goubert J-P, Ladurie ELR. La conquête de l'eau : l'avènement de la santé à l'âge industriel. Paris : Robert Laffont, 1986.

31. Csergo J. Liberté, égalité, propreté : la morale de l'hygiène au XIX ${ }^{\mathrm{e}}$ siècle. Paris : Albin Michel, 1988.

32. Canguilhem G. Le normal et le pathologique. Paris : Presses universitaires de France, 1972.

33. Elias N, Kamnitzer P. La civilisation des mœurs. Paris : Calmann-Lévy, 1973.

34. Foucault M. Naissance de la clinique. Paris : Gallimard, 2003.

35. Foucault M. Surveiller et punir. Paris : Gallimard, 1975.
36. Lichtenthaeler C, Meunier D. Histoire de la médecine. Paris : Fayard, 1978.

37. Starobinski J. Histoire de la médecine. Paris : Editions Rencontre, 1963.

38. Grmek MD. Préliminaires d'une étude historique des maladies. Annales, Economies, Sociétés, Civilisations 1969:1473-83.

39. Havelange C. Les figures de la guérison, $\mathrm{XVIII}^{\mathrm{e}}-\mathrm{XIX}{ }^{\mathrm{e}}$ siècles : une histoire sociale et culturelle des professions médicales au pays de Liège. Liège : Librairie Droz, 1990.

40. Sendrail M, Baudot G. Histoire culturelle de la maladie. Paris : Privat, 1981.

41. Porter D, Porter R. Patient's progress: doctors and doctoring in eighteenth-century England. Stanford: Stanford University Press, 1989.

42. Porter R. The patient's view. Theory and society 1985;14:175-98.

43. Porter R. The Cambridge history of medicine. Cambridge: Cambridge University Press, 2006.

44. Porter R. Flesh in the Age of Reason. New York: WW Norton \& Company, 2003.

45. Porter D. Health, civilization, and the state: a history of public health from ancient to modern times. Londres: Psychology Press, 1999.

46. Porter D. The history of public health and the modern state. Amsterdam: Rodopi, 1994.

47. Duffin J. Lovers and livers: Disease concepts in history. Toronto: University of Toronto Press, 2005.

48. Dosse F. L'histoire en miettes : des « Annales » à la « nouvelle histoire ». Paris : La découverte, 1987.

49. Prost A. Douze leçons sur l'histoire. Paris : Points, 2014.

50. Dewey J. Logique : la théorie de l'enquête. Paris : Presses Universitaires de France, 1967.

51. Durand G. Histoire de l'éthique médicale et infirmière : contexte socioculturel et scientifique. Montréal : PUM, 2000.

52. Goldenberg MJ. On evidence and evidence-based medicine: lessons from the philosophy of science. Soc Sc Med 2006;62:2621-32.

53. Le Breton $\mathrm{D}$. Le gène comme patient: une médecine sans sujet. Les Cahiers du Centre Georges Canguilhem 2007(1):15-31. 
54. Absil G, Vandoorne C, Harmant LF. L'écriture de nous, autobiographie collective contre les fictions sociales de stigmate. In: Constantopoulos C. (Ed.) Récits et fictions dans la société contemporaine. Pairs : Editions L'Harmattan, 2011:151-73.

55. Absil G. Le culte de saint Marcoul dans le diocèse de Liège $\left(\mathrm{XV}^{\mathrm{e}}-\mathrm{XVIII}{ }^{\mathrm{e}}\right.$ siècles) : approche de la représentation des écrouelles et des scrofuleux. Liège : Université de Liège, 1999.

56. Csordas TJ. Embodiment and experience: The existential ground of culture and self. Cambridge: Cambridge University Press, 1994.

57. de Certeau M. La possession de Loudun. Paris : Julliard, 1975.

58. Hage J. L'histoire culturelle : un «tournant mondial » dans l'historiographie ? Bulletin des bibliothèques de France 2009 [On-line] Disponible sur : http:// bbf.enssib.fr/consulter/bbf-2009-03-0104-004

59. Havelange C. De l'oeil et du monde : une histoire du regard au seuil de la modernité. Paris : Fayard, 1998.

60. Chartier R. La nouvelle histoire culturelle existe-telle ? Cahiers du Centre de Recherches Historiques 2003;31:13-24.

61. Pelaccia T, Paillé P. La recherche qualitative en pédagogie médicale : histoire, pratique et légitimité. Pédagogie Médicale 2011;12:179-92.
62. Rolfe G, Gardner L. Towards a geology of evidencebased practice - a discussion paper. Int J Nurs Stud 2006;43:903-13.

63. Chartier R. Au bord de la falaise. L'histoire entre certitudes et inquiétude. Paris : Albin Michel, 2009:37.

64. Foucault M. L'ordre du discours. Paris : Gallimard, 1971.

65. Rioux J-P, Sirinelli J-F. Pour une histoire culturelle. Paris : Seuil, 1997.

66. Prochasson C. Introduction : Autour de l'histoire culturelle. Les Cahiers du Centre de Recherches Historiques 2003 [On-line]. Disponible sur : http:// ccrh.revues.org/305

67. Werner M, Zimmermann B. Penser l'histoire croisée : entre empirie et réflexivité. Annales Histoire en sciences sociales 2003;1:7-36.

68. Bourdieu P. L'objectivation participante. Actes de la recherche en sciences sociales 2003;150:43-58.

Correspondance et offprints : Gaëtan ABSIL, Département des sciences de la santé publique, Laboratoire d'anthropologie sociale et culturelle, Université de Liège Sart Tilman B 23, 4000 Liège, Belgique. Mailto : gaetan.absil@ulg.ac.be 